Wellington $\mathrm{H}$. Ow

Nagoya Math. J.

Vol. 48 (1972), 57-65

\title{
$\Phi$-BOUNDED HARMONIC FUNCTIONS AND THE CLASSIFICATION OF HARMONIC SPACES
}

\author{
WELLINGTON H. OW
}

1. By a harmonic space we mean a pair $(X, H)$ where $X$ is a locally compact, non-compact, connected, locally connected Hausdorff space; and $H$ is a sheaf of harmonic functions defined as follows: Suppose to each open set $\Omega \subset X$ there corresponds a linear space $H(\Omega)$ of finitely-continuous real-valued functions defined on $\Omega$. Then $H=\{H(\Omega)\}_{\Omega}$ must satisfy the three axioms of Brelot (1) and in addition Axiom 4 of Loeb (4): 1 is $H$-superharmonic in $X$.

Denote by $\Phi(t)$ a nonnegative real-valued function defined on $[0, \infty)$. We stress that except for the condition $\Phi(t) \geqslant 0$ nothing is required of $\Phi(t)$ such as continuity and measurability. A harmonic function $u$ on $X$ (when $H$ is well-understood we simply refer to $X$ itself as the harmonic space) is called $\Phi$-bounded if the composite function $\Phi(|u|)$ possesses a harmonic majorant on $X$. The notion of $\Phi$-boundedness is due to Parreau (9) who considered the special case of an increasing, convex $\Phi$. Later Nakai (6), using general $\Phi$, completely determined the class $O_{H \Phi}$ of Riemann surfaces for which every $\Phi$-bounded harmonic function reduces to a constant. Recently Ow (8) considered the classification of harmonic spaces with respect to $\Phi$-bounded harmonic functions using a stronger assumption that Loeb's Axiom 4; namely it was assumed that $1 \in H$.

Since the case $1 \in H$ has already been considered, as mentioned above, throughout this paper we will make the following assumption:

$$
1 \notin H \text {. }
$$

This condition occurs, for example, in the study of the harmonic space of solutions of the elliptic partial differential equation $\Delta u=P u$, where $P \not \equiv 0$ is a nonnegative function on a manifold $X$.

The main object of this paper is to show that in view of the con-

Received August 16, 1971. 
dition $1 \notin H$, the assumed existence of a harmonic function $u$ on $X$ with positive infimum is essential in the classification of harmonic spaces with respect to $\Phi$-boundedness. Furthermore, it is shown that is sometimes necessary to further assume that the function $u$ above is bounded in order to obtain inclusion relations similar to those in (8). Before proceeding further it is necessary to give some preliminary results.

2. If $K$ is a compact subset of $X$ and $E$ the family of all regular regions $\Omega$ (cf. (4)) containing $K$ then by a theorem of Loeb (4), $E$ is an exhaustion of $X$. We will always assume that $X$ is countable at the ideal boundary and therefore there exists a countable exhaustion of $X$ by regular regions $\left\{\Omega_{n}\right\}_{1}^{\infty}$ such that $\bar{\Omega}_{n} \subset \Omega_{n+1}$ and $X=\bigcup_{n=1}^{\infty} \Omega_{n}$.

We now state some results of Loeb-Walsh (5) using their terminology. Let $e$ be the greatest $H$-harmonic minorant of 1 and assume that $e \neq 0$. Denote by $H B=H B(X)$ the Banach lattice of bounded functions in $H$. Note that $H B \neq\{0\}$. Let $X^{*}$ be the $H B$-compactification of $X, \Gamma=X^{*}-$ $X$, and

$$
\Delta=\left\{t \in \Gamma \mid e(t)=1 \text { and } f \wedge_{H} g(t)=f \wedge g(t) \text { for all } f, g \in H B\right\} .
$$

It is shown in (5) that $\Delta$ is regular for the Dirichlet problem and is also equivalent to the harmonic boundary of Constantinescu-Cornea (3). Also it is shown in (5) that the restriction mapping of $H B$ onto $C(\Delta)$ is an isometric isomorphism which preserves positivity and lattice operations.

3. If $\Omega$ is a subregion of $X$ then we will say that $\Omega \notin S O_{H B}$ provided $\Omega$ contains a neighborhood of some point $p \in \Delta$. We then have the following generalization of the well-known two-domain criterion for Riemann surfaces (cf. e.g. (10)):

LEMMA 1. There exists at least $k \geqslant 1$ disjoint regions $\Omega_{i} \subset X$ with $\Omega_{i} \notin S O_{H B}$ if and only if $\operatorname{dim} H B \geqslant k$.

Proof. It follows from the definition that if there exist at least $k \geqslant 1$ disjoint regions $\Omega_{i} \notin S O_{H B}$ then $\Delta$ contains at least $k$ points and hence $\operatorname{dim} H B=\operatorname{dim} C(\Delta) \geqslant k$. Conversely suppose $\operatorname{dim} H B \geqslant k$. Then there exists at least $k$ points $p_{j} \in \Delta$. Let $f$ be a Wiener function, i.e. a bounded, continuous, harmonizable function on $X$ (cf. (10)) such that $f\left(p_{j}\right)=j . \quad$ Set $G_{j}^{*}=\left\{p \in X^{*} \mid j-\frac{1}{2}<f(p)<j+\frac{1}{2}\right\}$ and $G_{j}=G_{j}^{*} \cap X$. Then $G \notin S O_{H B}$ and the $G_{j}$ are disjoint. This completes the proof. 
4. As an immediate consequence of a result of ConstantinescuCornea (cf. (3), p. 32) the following maximum principle of Nakai (10) is also valid for harmonic spaces:

LEMMA 2. Let $\Omega$ be a subregion of $X$ and $s$ a superharmonic function on $\Omega$ bounded from below. If

$$
\liminf _{z \in \Omega, z} s(z) \geqslant 0
$$

for every point $p \in(\Delta \cap \bar{\Omega}) \cup \partial \Omega$ then $s \geqslant 0$ on $\Omega$. Here $\bar{\Omega}$ means the closure of $\Omega$ in $X^{*}$ while $\partial \Omega$ denotes the boundary of $\Omega$ relative to $X$.

5. Denote by $H \Phi=H \Phi(X)$ the family of all $\Phi$-bounded harmonic functions on $X$ and by $O_{H \Phi}$ the totality of harmonic spaces on which every $\Phi$-bounded harmonic function reduces to a constant. Similarly denote by $H P=H P(X), H B=H B(X)$ the class of functions on $X$ which are nonnegative harmonic and bounded harmonic, respectively; and by $O_{H P}$ (resp. $O_{H B}$ ) the class of harmonic spaces $X$ for which the class $H P$ (resp. $H B$ ) consists only of constants. We define

$$
\bar{d} \Phi=\limsup _{t \rightarrow \infty} \Phi(t) / t \quad \text { and } \quad \underline{d} \Phi=\liminf _{t \rightarrow \infty} \Phi(t) / t .
$$

Suppose that there exists a positive harmonic function on $X$ with positive infimum. We then note first that if $\Phi$ is bounded on $[0, \infty)$ then any nonconstant harmonic function on $X$ is a nonconstant $H \Phi$-function, and consequently, $O_{H \Phi}$ consists only of trivial harmonic spaces. On the other hand if $\Phi(t)$ is completely unbounded on $[0, \infty)$, i.e. if $\Phi(t)$ is not bounded in any neighborhood of any point of $[0, \infty)$ then $O_{H \Phi}$ must consist of all harmonic spaces. Having dispensed with these cases we now prove a result similar to one obtained for Riemann surfaces by Nakai (6).

THEOREM 1. Assume there exists a bounded harmonic function $u_{0}$ on $X$ with $\inf _{X} u_{0}>0$. Then if $\Phi$ is not bounded nor completely unbounded on $[0, \infty), O_{H \Phi}=O_{H P}$ (resp. $\left.O_{H \Phi}=O_{H B}\right)$ provided that $\bar{d}(\Phi)$ is finite (resp. infinite).

A proof of Theorem 1 will be given in section 7. Using stronger assumptions on $\Phi$, Chow-Glasner (2) have obtained results similar to Theorem 1 in their investigation on $\Phi$-bounded solutions of $\Delta u=P u$, $P \geqslant 0$, on Riemannian manifolds. Namely they assume that $\Phi$ is convex, positive, and increasing. 
6. The next theorem shows the effect of omitting either the boundedness condition or the condition $\inf _{X} u_{0}>0$ as was required of the function $u_{0}$ in Theorem 1 .

THEOREM 2. Assume $\Phi$ is not bounded nor completely unbounded on $[0, \infty)$.

a) If $\bar{d}(\Phi)<\infty$ then $O_{H P} \subset O_{H \Phi}$.

b) If $\bar{d}(\Phi)<\infty$ and if there exists an HP-function $u_{1}$ with $\inf _{X} u_{1}>0$, then $O_{H \Phi} \subset O_{H P}$. But if $\bar{d}(\Phi)<\infty$, if there exists a nonconstant $H P$ function, and if $u \in H P$ implies $\inf _{X} u=0$, then $O_{H \Phi} \subset O_{H P}$ is not necessarily true.

c) If $\bar{d}(\Phi)=\infty$ then $O_{H B} \subset O_{H \Phi}$.

d) If $\bar{d}(\Phi)=\infty$ and there exists an HP-function $u_{0}$ such that $u_{0}$ is bounded and $\inf _{X} u_{0}>0$, then $O_{H \Phi} \subset O_{H B}$. However, if $\bar{d}(\Phi)=\infty$ and every HP-function $u$ is either unbounded or $\inf _{X} u=0$ then $O_{H \Phi} \subset O_{H B}$ is not necessarily true.

A proof of Theorem 2 appears in section 8. The existence of $u_{1}$ is also considered by Schiff (12) in the special case concerning solutions of $\Delta u=P u$ on a Riemann surface.

7. Proof of Theorem 1. First assume $\bar{d}(\Phi)<\infty$. Then there exists a $c>0$ such that $\Phi(t) \leqslant c t$ for $t \geqslant t_{0}$. If $u$ is a nonconstant $H P$-function on $X$ then for a suitable constant $k>0$ the function $v=u+k u_{0}$ is a nonconstant $H \Phi$-function, and so $O_{H \Phi} \subset O_{H P}$.

Conversely if $u$ is a nonconstant $H \Phi$-function on $X$ then there exists an $H P$-function $v$ on $X$ with $\Phi(|u|) \leqslant v$ on $X$. Since $1 \notin H, v$ is nonconstant. Hence $O_{H P} \subset O_{H \Phi}$, completing the first part of the proof.

Now consider the case where $\bar{d}(\Phi)=\infty$. Suppose $u$ is a nonconstant $H B$-function on $X$. By hypothesis $\Phi$ is bounded in some interval $(a, b)$ $\subset[0, \infty)$ within which $\Phi(t) \leqslant c=$ const. Then for suitable constants $c_{1}$ and $c_{2}$ the range of $v=c_{1} u+c_{2} u_{0}$ is contained in $(a, b)$, and consequently $O_{H \Phi} \subset O_{H B}$.

Conversely, if we assume $u$ is a nonconstant $H \Phi$-function on $X$ then there exists an $H P$-function $v$ on $X$ such that $\Phi(|u|) \leqslant v$ on $X$. If $v$ is bounded we are done. If $u$ is not bounded then following the approach of Nakai (6) we show that $X \notin O_{H B}$. Suppose to the contrary that 
$X \in O_{H B}$. Then $\bar{d}(\Phi)=\infty$ implies that there is a strictly increasing sequence $\left\{t_{n}\right\}_{1}^{\infty}$ of positive numbers for which $\lim _{n} t_{n}=\infty, \lim _{n} t_{n} / \Phi\left(t_{n}\right)=0$ and

$$
G_{n}=\left\{p \in X|| u(p) \mid<t_{n}\right\} \neq \phi .
$$

Then $G_{1} \subset G_{2} \subset \cdots$ and $X=\cup_{1}^{\infty} G_{n}$. Now $G_{n} \notin S O_{H B}$ for all sufficiently large $n$. For if not, consider the function $a_{n} v-|u|$ where $a_{n}=t_{n} / \Phi\left(t_{n}\right)$. Then $a_{n} v-|u|$ is superharmonic, bounded from below on $G_{n}$, and nonnegative on $\partial G_{n}$. Hence $G_{n} \in S O_{H B}$ implies $a_{n} v-|u| \geqslant 0$ on $G_{n}$ by Lemma 2. Since $a_{n} \rightarrow 0$ and $G_{n} \uparrow X$ we have $u \equiv 0$ on $X$, a contradiction. Hence $G_{n} \notin S O_{H B}$ for $n \geqslant n_{1}$, say, and so we may as well assume

$$
G_{n} \notin S O_{H B}, \quad n=1,2, \ldots
$$

If $G_{n}-\bar{G}_{1} \notin S O_{H B}$ for some $n>1$ then by Lemma $1, X \notin O_{H B}$, contradicting our original assumption. Hence

$$
G_{n}-\bar{G}_{1} \in S O_{H B}, \quad n=2,3, \cdots
$$

The function $w_{n}=a_{n} v+r_{1}-|u|$ is superharmonic, bounded from below on $G_{n}$ as well as $G_{n}-\bar{G}_{1}$. Also $w_{n} \geqslant 0$ on $\partial G_{n}$. Since $G_{n}-\bar{G}_{1} \in S O_{H B}$ this implies $w_{n} \geqslant 0$ on $G_{n}$, i.e.

$$
|u| \leqslant a_{n} v+r_{1}
$$

on $G_{n}$. Hence $|u| \leqslant r_{1}$ on $X$, contradicting our assumption $X \in O_{H B}$. Hence $X \notin O_{H B}$, completing the proof.

8. Proof of Theorem 2. Parts a) and c) are proved exactly as in the proof of Theorem 1 since the function $u_{0}$ is not involved. The first part of b) follows exactly as in Theorem 1 since only the condition $\inf _{X} u_{0}>0$ is used there. For the second half of $b$ ) consider the following example:

Example 1. Define $\Phi(t)=1 / t^{2}, t>0 ; \Phi(0)=0$. Then $\bar{d}(\Phi)=0<\infty$. Also for any harmonic function $u$, either $u \in H P$ or $-u \in H P$ or $u$ assumes the value 0 on $X$. In either case $\inf _{X}|u|=0$. It follows that $\Phi(|u|)$ has no $H P$-majorant on $X$, i.e. $O_{H \Phi} \not \subset O_{H P}$.

The first assertion in d) constitutes part of Theorem 1. For the second part of $d$ ) consider the following example in the complex plane $C$ :

EXAMPle 2. Let $X=\{z \in C|0<| z \mid<1\}$ and $H$ consist of all solutions of the elliptic partial differential equation $\Delta u=P u$ on $X$, where $P=4 /|z|^{2}$ 
and $\Delta=\partial^{2} / \partial x^{2}+\partial^{2} / \partial y^{2}, z=x+i y$. Note that $u_{1}=|z|^{2} \in H, u_{2}=1 /|z|^{2} \in H$, but $1 \notin H$. Since $u_{1} \in H$ the equation $\Delta u=P u$ has no bounded solution $u$ with $\inf _{X} u>0$. However, $u_{2}$ is an unbounded positive solution with $\inf _{X} u_{2}>0$. Let $\Phi$ be a nonnegative real-valued function on $[0, \infty)$ which is unbounded at the points $1 / n, n=1,2, \cdots$, and also at the points $n$, $n=2,3, \cdots$. Then since any member of $H$ must either be unbounded on $X$ or have zero infimum in its absolute value on $X$, it follows that there are no nonconstant $\Phi$-bounded solutions on $X$, i.e. $O_{H \Phi} \not \subset O_{H B}$. This completes the proof.

9. A harmonic function $\mathrm{u}$ on $X$ is called essentially positive if $u$ can be represented as a difference of two $H P$-functions on $X$, or equivalently, if $|u|$ has a harmonic majorant on $X$. Let $H P^{\prime}(X)$ be the vector lattice of essentially positive harmonic functions on $X$ with lattice operations $\vee$ and $\wedge$, where for two functions $u$ and $v$ in $H P^{\prime}(X)$ we denote by $u \vee v($ resp. $u \wedge v$ ) the least harmonic majorant (resp. the greatest harmonic minorant) of $u$ and $v$. Clearly $H P(X) \subset H P^{\prime}(X)$.

For any $u \in H P(X)$ we define the function $B u$ by

$$
B u(p)=\sup \{v(p) \mid v \in H B(X), v \leqslant u \text { on } X\} .
$$

If $u \in H P^{\prime}(X)$ we define $B u=B u_{1}-B u_{2}$ where $u=u_{1}-u_{2}$ and $u_{1}, u_{2} \in H P(X)$. An $H P^{\prime}$ function $u$ is called quasi-bounded (resp. singular) if $B u=$ $u$ (resp. $B u=0$ ). We denote the class of quasi-bounded (resp. singular) functions on $X$ by $H B^{\prime}(X)$ (resp. $H P^{\prime \prime}(X)$ ). We then have the direct decomposition

$$
H P^{\prime}(X)=H B^{\prime}(X)+H P^{\prime \prime}(X) .
$$

Quasi-bounded and singular harmonic functions as well as the decomposition were introduced by Parreau (9). We now give relations between the classes $H \Phi, H B^{\prime}$, and $H P^{\prime}$. The following theorem is similar to that obtained by Nakai (7) for Riemann surfaces:

THEOREM 3. Assume there exists an HP-function $u_{1}$ on $X$ with $\inf _{X} u_{1}>0$.

a) If $\underline{d}(\Phi)>0$ then $H \Phi(X) \subset H P^{\prime}(X)$.

b) If, however, $\underline{d}(\Phi)=0$ then $H \Phi(X) \subset H P^{\prime}(X)$ is not necessarily true.

Proof. To prove a) we set $d(\Phi)=2 \mathrm{c}>0$ and choose $t_{0} \in(0, \infty)$ so 
that $\Phi(t)>c t$ for $t>t_{0}$. If $u \in H \Phi(X)$ then $\Phi(|u|)$ has a harmonic majorant $v$ on $X$. It follows that for a suitable constant $k>0$ we have

$$
v+c k u_{1} \geqslant \Phi(|u|)+c t_{0} \geqslant c|u|
$$

on $X$ and $|u|$ possesses a harmonic majorant on $X$; so $u \in H P^{\prime}(X)$, thereby proving a).

To prove b) we consider the following example in the plane:

Example 3. As in Example 2 let $X=\{z \in C|0<| z \mid<1\}$ and $H$ consist of solutions of $\Delta u=P u$, with $P=4 /|z|^{2}$. Recall that $u_{1}=1 /|z|^{2}$ is an $H P$-function on $X$ with $\inf _{X} u_{1}>0$. Consider the function $u$ in $H$ given by

$$
u(z)=\cos (\sqrt{5} \theta) / r^{3}, \quad z=r e^{i \theta},
$$

and also the function $\Phi(t)=\max (\log t, 0)$ on $[0, \infty)$. Then $\underline{d}(\Phi)=0$, $u \in H \Phi(X)$ but $u \notin H P^{\prime}(X)$. This completes the proof.

The following theorem of Nakai (7) is also valid for harmonic spaces:

THEOREM 4. If $\bar{d}(\Phi)=\infty$ then $H \Phi(X) \cap H P^{\prime}(X) \subset H B^{\prime}(X)$.

Proof. For $u \in H \Phi(X) \cap H P^{\prime}(X)$ there exists an $H P$-function $v$ on $X$ with $\Phi(|u|) \leqslant v$. Define $M u=u \vee 0+(-u) \vee 0$. Since $B$ commutes with the operations $M, \vee$, and $\wedge$ we need only show

$$
B M u=M u .
$$

Since $\bar{d}(\Phi)=\infty$ there is an increasing sequence $\left\{t_{n}\right\}_{1}^{\infty}$ of positive numbers with $\Phi\left(t_{n}\right)>0$ and $a_{n}=t_{n} / \Phi\left(t_{n}\right) \rightarrow 0$. Setting $G_{n}=\left\{p \in X|| u(p) \mid<t_{n}\right\}$ we have $G_{n} \uparrow X$. Let $\left\{\Omega_{m}\right\}$ be an exhaustion of $X$. Let $w_{m}$ be harmonic on $\Omega_{m} \cap G_{n}$ with $w_{m} \mid\left(\partial \Omega_{m}\right) \cap G_{n}=\min \left(M u-B M u, t_{n}\right)$ and $w_{m} \mid\left(\partial G_{n}\right) \cap \bar{\Omega}_{m}=$ 0 . Here the values of $w_{m}$ on $\partial\left(\Omega_{m} \cap G_{n}\right)$ need only be prescribed at the points regular for the Dirichlet problem. If we further define $w_{m} \mid\left(\Omega_{m}\right.$ $\left.-G_{n}\right)=0$ then $w_{m}$ is subharmonic on $\Omega_{m}$, and hence

$$
w_{m} \geqslant w_{m+1}
$$

on $\Omega_{m}$ (cf. Loeb-Walsh (5)). Also let $w_{m}^{\prime}$ be harmonic on $\Omega_{m}$ with boundary values $\quad w_{m}^{\prime} \mid\left(\partial \Omega_{m}\right) \cap G_{n}=\min \left(M u-B M u, t_{n}\right)$ and $w_{m}^{\prime} \mid\left(\partial \Omega_{m}-G_{n}\right)=0$. Then $\left\{w_{m}^{\prime}\right\}$ is a bounded sequence and $0 \leqslant w_{m}^{\prime} \leqslant M u-B M u, m=1,2, \cdots$. It follows from a theorem of Loeb-Walsh (5) that if $\Omega \subset X$ is a region 
and the family $T=\{h \in H(\Omega) \mid 0 \leqslant h\}$ is bounded then $T$ is equicontinuous on $\Omega$. Consequently by the Arzelà-Ascoli theorem $T$ is a normal family. Hence $\left\{w_{m}^{\prime}\right\}$ has a convergent subsequence with limit function $w^{\prime}$. We obtain $0 \leqslant B w^{\prime} \leqslant B(M u-B M u)=0$. Since $w^{\prime}$ is bounded and nonnegative,

$$
w^{\prime} \equiv B w^{\prime} \equiv 0
$$

on $X$. In addition $w_{m}^{\prime} \geqslant w_{m} \geqslant 0$ implies

$$
\lim _{m} w_{m}=0
$$

on $X$. Now on $\left(\partial \Omega_{m}\right) \cap G_{n}$ we have $|u| \leqslant t_{n}$ and $|u| \leqslant M u=B M u+$ $(M u-B M u)$. Hence on $\left(\partial \Omega_{m}\right) \cap G_{n},|u|-B M u \leqslant \min \left(M u-B M u, t_{n}\right)=$ $w_{m}$. On $\partial G_{n},|u|=t_{n}=a_{n} \Phi(|u|) \leqslant a_{n} v$, and so

$$
|u| \leqslant a_{n} v+B M u+w_{m}
$$

on $\partial\left(\Omega_{m} \cap G_{n}\right)$ and hence on $\Omega_{m} \cap G_{n}$. Upon letting $m \rightarrow \infty$ and then $n \rightarrow \infty$ we obtain

$$
|u| \leqslant B M u
$$

on $X$. Since $M u$ is the least harmonic majorant of $|u|$ on $X$ we must have $M u \leqslant B M u$ and hence $B M u=M u$ as was to be shown. This completes the proof.

Remark. Note that the existence of a function $u_{1}$ as in Theorem 3 is not required here.

Upon combining Theorem 3 and Theorem 4 we have the following

Corollary. Assume there exists an $H P$-function $u_{1}$ on $X$ with $\inf _{X} u_{1}>0$. Then if $\bar{d}(\Phi)=\infty$ and $\underline{d}(\Phi)>0$, we have $H \Phi(X) \subset H B^{\prime}(X)$.

\section{REFERENCES}

[1] M. Brelot, Lectures on potential thèory, Tata Inst. Fund. Res., Bombay, 1960.

[2] K. Chow and M. Glasner, Bounded in the mean solutions of $\Delta u=P u$ on Riemannian manifolds, Proc. Amer. Math. Soc. 26 (1970), 261-265.

[ 3 ] C. Constantinescu and A. Cornea, Compactifications of harmonic spaces, Nagoya Math. J. 25 (1965), 1-57.

[4] P. A. Loeb, An axiomatic treatment of pairs of elliptic differential equations, Ann. Inst. Fourier, Grenoble 16 (1966), 167-208.

[5] P. A. Loeb and B. Walsh, A maximal regular boundary for solutions of elliptic differential equations, Ann. Inst. Fourier, Grenoble 18 (1968), 283-308.

[6 ] M. Nakai, $\Phi$-bounded harmonic functions and classification of Riemann surfaces, Pacific J. Math. 15 (1965), 1329-1335. 
[ 7 ] M. Nakai, On $\Phi$-bounded harmonic functions, Ann. Inst. Fourier 16 (1966), $145-157$.

[ 8 ] W. H. Ow, Wiener's compactification and $\Phi$-bounded harmonic functions in the classification of harmonic spaces, Pacific J. Math. (to appear).

[9] M. Parreau, Sur les moyennes des fonctions harmoniques et analytiques et la classification des surfaces de Riemann, Ann. Inst. Fourier (Grenoble) 3 (1951), 103-197.

[10] L. Sario and M. Nakai, Classification theory of Riemann surfaces, SpringerVerlag, Berlin-Heidelberg-New York, 1970.

[11] J. L. Schiff, $\Phi$-bounded solutions of $\Delta u=P u$ on a Riemann surface, (to appear).

Michigan State University 